\title{
Intensity of the underlying figural process
}

TEODOR KÜNNAPAS

PSYCHOLOGICAL LABORATORIES, UNIVERSITY OF STOCKHOLM, SWEDEN

A simple scaling technique is described by means of which proportions of frequencies may be transformed to scale values representing the intensity of the perceptual process. This technique is applied to data from experiments on directly observable figural fluctuations. The intensity of the underlying figural process is defined by a sine function with amplitude damping. Very good agreement between theoretical and empirical values demonstrates the applicability of the proposed model.

In recent years the well-known phenomenon of reversals of different ambiguous visual patterns has been investigated in a number of investigations (Kunnapas, 1957; Cohen, 1959; Oyama, 1960; Torii, 1960; Howard, 1961; Spitz \& Lipman, 1962; Heath, Ehrlich, \& Orbach, 1963; Orbach, Ehrlich, \& Vainstein, 1963; Thetford, 1963; Kolers, 1964; Olson \& Orbach, 1966; Orbach, Zucker, \& Olson, 1966).

All these studies have called attention to the reversals which are directly observable by the subject. These immediately observable fluctuations may be called figural fluctuations. The approach of the investigators to these fluctuations was only to record the response phases of the fluctuations, to compute the average rate of fluctuations, to study the effect of repeated or prolonged observation on this rate, the effect of area, hue, brightness, orientation of the figure and intrafigural angle in a reversible pattern on the figural fluctuation, and so on.

The perceptual process underlying the observable fluctuations will be called figural process. It is not observable and is therefore unknown, but it appears in observable fluctuations. These observable fluctuations are regarded as a function of the individual's underlying figural process. The above-mentioned studies do not investigate the underlying figural process.

The purpose of the present investigation was to study the intensity of the underlying figural process and to measure it in psychological units, applying an indirect scaling technique.

\section{Previous work}

\section{THE SCALING METHOD}

The fundamental principles of indirect psychological measurement were introduced by Thurstone (1927), who developed the concept of discriminal dispersion. In more recent years indirect scaling methods have been developed for measurement of unobservable processes (Bjorkman, 1958).

The present author has used a similar approach in an investigation of a reversible visual pattern (Künnapas, 1961). A quantitative indirect method was developed to obtain measures of the intensity difference between two conflicting perceptual processes, and this intensity difference could be described as a periodic function of time.

A similar method was used by Ekman and Lindman (1962) in a study of perceptual fluctuations at liminal scotopic stimulation and in an investigation of visual after-image intensity as a function of time (Ekman \& Lindman, 1964).

\section{The metric model}

The metric model is described in some detail in the previous report (Künnapas, 1961). The fundamental features of the model are illustrated in Fig. 1.

Each stimulus when presented to a subject gives rise to a process which has some value on the continuum of intensity. An ambiguous stimulus pattern, corresponding to the two different parts of the whole figure, a and $b$, would excite two processes, the intensities of which will be denoted $s_{a}$ and $s_{b}$, respectively. It is assumed that these two processes are conflicting and that the intensity of the resulting figure process is equal to the difference between the intensities of the component processes $\left(s_{a}-s_{b}\right)$.

If the stimulus pattern is presented a large number of times, the differences themselves will form a distribution on the continuum of intensity. Consider in Fig. 1 the point $t$ on the time axis. The intensity differences $y$ of two conflicting perceptual processes has different values in different trials, and this variation is represented by the frequency distribution, the mean of which is $y_{t}$.

The curve in Fig. 1 represents the average intensity

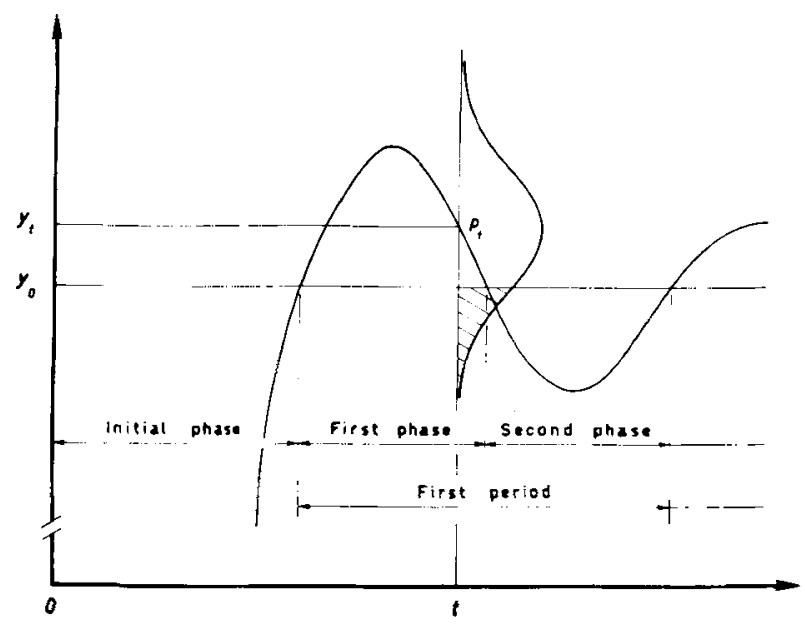

Fig. 1. The metric model. For explanation, see text. 
difference between two conflicting perceptual processes, evoked by stimulation with an ambiguous pattern, as a function of time. After a very short time, usually a fraction of a second, from the onset of stimulation (the zero point of the time axis) the intensity begins to grow rapidly and reaches a level that is fairly constant except for a periodic variation over time. Our primary concern will be with this systematic trend of variation.

In addition to the systematic trend, however, a random variation occurs over time and between trials. This fluctuation constitutes the dispersion of differences around the mean, which we shall use to obtain measures of the systematic trend.

The horizontal line drawn through $y_{0}$ represents the threshold which separates the two alternative responses from one another. If the first phase is marked as positive, the second phase is negative. The difference between the intensities of two conflicting perceptual processes $\left(s_{a}-s_{b}\right)$ is positive whenever process $a$ is more intense than process $b$, and negative whenever $b$ has a greater intensity than a. The unshaded portion of the frequency distribution of differences represents the proportion $p_{t}$ of all the trials in which a perception of the first response phase is reported by the subject at time $t$ after the onset of stimulation.

\section{Scale construction}

The experimental reports of subjects are in the form of "figure a" and "figure b" in temporal sequence, and the frequency of reported alternative figures are converted into proportions $\mathrm{p}_{\mathrm{t}}$. The scaling problem consists in obtaining scale values $y_{t}$ from the proportions. To do this we have to specify the general model by introducing certain assumptions.

If we assume that the frequency distribution of differences shown in Fig. 1 around the mean $y_{t}$ is normal, $y_{t}$ may be measured in terms of the standard deviation $\sigma_{a-b}$ of this distribution and with $y_{0}$ as an arbitrary zero point. By definition of the normal deviate

$$
z_{t}=\left(y_{0}-y_{t}\right) / \sigma(a-b)_{t} !
$$

where $\sigma(a-b)_{t}$ is the standard deviation of $y$ at time $t$. Solving for $y_{t}$,

$$
\mathrm{y}_{\mathrm{t}}=\mathrm{y}_{\mathrm{o}}-\mathrm{z}_{\mathrm{t}} \sigma_{(\mathrm{a}-\mathrm{b})_{\mathrm{t}}}
$$

and transforming the scale so that $y_{0}$ is the arbitrary zero point, we obtain the new scale,

$$
y_{\dagger}^{\prime}=z_{\dagger} \sigma_{(a-b)_{\dagger}^{\prime}}
$$

where the sign of $z_{t}$ is positive if the proportion of part-figure $a$ is greater than that of part-figure $b$, and negative if the proportion of part-figure $b$ is greater than that of figure $a$. By definition, $z_{t}=0$, if $p_{a}-p_{b}=0$.

The assumption of normality is probably not critical and moderate deviations from this condition are not

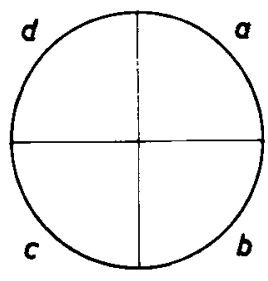

A

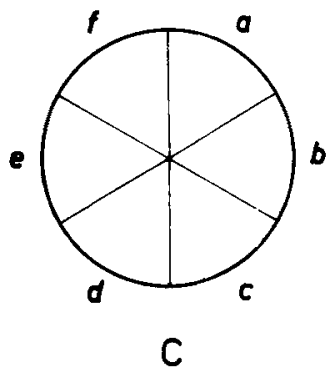

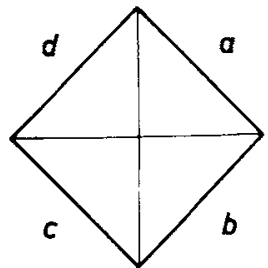

B

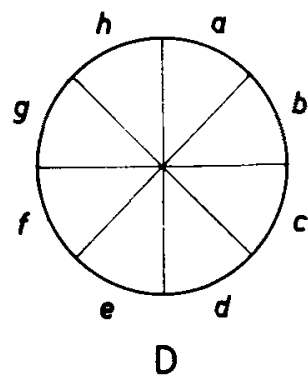

Fig. 2. The four stimulus patterns $(A, B, C, D)$ used in experiments. On the circle with six sectors (C), e.g., the sectors a, c, and $e$ form the "right," and the sectors $b$, $d$, and $f$ the "left" part-figure.

likely to distort the scale seriously.

The additional assumption will be that $\sigma(a-b)_{t}$ is constant for different values of $y_{t}$. Under these circumstances, ${ }^{\sigma}(a-b)_{t}$ may be used as the constant unit of measurement $\left(\sigma(a-b)_{t}=1\right)$, so that the interval scale is defined by the normal deviate,

$$
y_{t}^{\prime}=z_{t} \text {. }
$$

The assumption of constant variation may or may not be true. Several investigations with direct methods have shown that a measure of uncertainty on the subjective continuum tends to increase with the subjective magnitude (Stevens, 1957; Ekman, 1956, 1959), and recent comparative studies of direct and indirect methods indicate a linear relationship between interindividual variation and mean scale value (Ekman \& Künnapas, 1962a, b, 1963). Neither of these two cases is directly comparable to the present situation, but the possibility must be considered that the assumption of a constant variation is erroneous. If $\sigma(\mathrm{a}-\mathrm{b})_{\mathrm{t}}$ increases over time, there will result an amplitude damping, which is at least in part an artifact. Further experiments are being conducted to study this question.

\section{EXPERIMENTS}

\section{Stimulus maferial}

Four ambiguous patterns were used as stimulus material: three circles with four, six and eight sectors, respectively, and a square with four triangles (Fig. 2). All stimulus patterns were contour figures with black 
Table 1. Individual parameters for the left and right part-figures

\begin{tabular}{|c|c|c|c|c|c|c|}
\hline Subject & Part-figure & $a$ & A & 9 & $\mathrm{p}$ & $r$ \\
\hline \multirow[t]{2}{*}{$T$} & Left & 1.50 & 1.10 & 0.37 & 177.3 & -0.004 \\
\hline & Right & 1.48 & 0.88 & 0.39 & 196.0 & -0.016 \\
\hline \multirow[t]{2}{*}{2} & Left & 0.94 & 1.99 & 0.49 & 241.0 & -0.014 \\
\hline & Right & 0.94 & 2.15 & 0.50 & 245.8 & -0.016 \\
\hline \multirow[t]{2}{*}{3} & Left & 1.54 & 0.83 & 0.72 & 138.0 & 0.029 \\
\hline & Right & 1.42 & 1.70 & 0.66 & 173.0 & 0 \\
\hline \multirow[t]{2}{*}{4} & Left & 2.20 & 0.80 & 0.33 & 209.0 & 0.015 \\
\hline & Right & 2.14 & 0.38 & 0.34 & 240.0 & -0.017 \\
\hline \multirow[t]{2}{*}{5} & Left & 1.90 & 0.46 & 0.07 & 187.6 & -0.037 \\
\hline & Right & 1.85 & 0.50 & 0.30 & 192.5 & 0 \\
\hline
\end{tabular}

lines on white background, presented by means of a projector, one slide being used for each pattern. Thus the sectors did not differ in brightness or color. In order to avoid the preference of the main verticalhorizontal direction as compared with tilted positions, the lines between the sectors were drawn so that all sectors were seen in tilted positions, as shown in Fig. 2. One half of the sectors formed the right part-figure, the others the alternative left part-figure. In the case of the circle with six sectors, for example, the sectors $a, c$ and $e$ form the "right," and the sectors $b, d$ and $f$ the "left" part-figure (Fig. 2C).

\section{Apparafus}

The slides with the stimulus figures were projected on a semi-transparent screen, one at a time. The size of the figure on the screen was $5 \mathrm{~cm}$ in diameter or in diagonal (pattern B).

The subject reported the fluctuation of both alternating part-figures by depressing two keys: the right-hand key for the right part-figure and the left-hand key for the left part-figure. These keys were connected to an electric writing unit which registered the duration of both response phases as lines of varying length on a moving paper strip.

The writing unit started automatically as soon as the stimulus pattern was visible to the subject on the screen, and this starting time of an observation trial was automatically marked on the moving paper strip.

\section{Procedure}

The subjects viewed the stimulus figure binocularly at a distance of $100 \mathrm{~cm}$. They were instructed to fixate the center of the pattern throughout the observation period, to press the right-hand or left-hand key as soon as they saw the corresponding part-pattern as a figure and keep pressing as long as that figure was seen. They were not to press the keys when no figure or only an undefined figure was seen. They were also instructed to report verbally which of the two alternating part-figures appeared as the first figure and to take a passive attitude and not to try to influence shifting.

One hundred trials were run for each of four stimulus patterns. The first 30 trials were considered as preliminary, and the last 70 were taken into account in treating the data. In each trial the time was recorded for the initial phase and for, at least, the first three subsequent response phases of both alternating partfigures. Between trials the subject rested $30 \mathrm{sec}$.

The stimulus patterns were rotated in blocks of 10 trials. The experiments were conducted in several sessions with each subject.

\section{Subjects}

Five students of psychology took part in the experiments. The subjects had acquired a certain practice in the observation of the reversals during the laboratory course in experimental psychology. In the preliminary trials they were made familiar with the experimental situation. They had normal visual acuity.

\section{Treafment of records}

The graphic records of each trial were examined at times $0.1,0.2,0.3,0.4 \mathrm{sec}$. and so on, where time 0 denotes the moment when the stimulus figure was first presented on the screen. The frequencies of reported part-figures were found for all these points on the time axis and were then transformed to proportions. By Equation (4) the corresponding $z$-values were found.

\section{EQUATION}

The general appearance of the data and their recurring character suggests a sine function with amplitude damping. In the previous report (Künnapas, 1961) this function is defined by the equation

$$
y_{\uparrow}=A e^{-q \dagger} \sin \left(p e^{r t_{+}}\right) \text {. }
$$

The first factor, $A e^{-q t}$, denotes the amplitude of the intensity of the figural process, where A expresses the initial amplitude and $q$ its damping with the time. The second temporal factor, sin $\left(\mathrm{pe}^{\mathrm{rt}} \mathrm{t}\right)$, denotes the periodicity of the process in temporal sequence as a

Table 2. Individual parameters for each stimulus pattern.

\begin{tabular}{|c|c|c|c|c|c|c|}
\hline Subject & Pattern & $a$ & A & $q$ & $P$ & $r$ \\
\hline \multirow[t]{4}{*}{1} & A & 1.33 & 1.35 & 0.30 & 198.6 & -0.019 \\
\hline & $B$ & 1.35 & 1.47 & 0.32 & 206.4 & -0.003 \\
\hline & $C$ & 1.60 & 1.48 & 0.28 & 172.0 & -0.005 \\
\hline & D & 1.73 & 1.24 & 0.44 & 176.0 & 0.001 \\
\hline \multirow[t]{4}{*}{2} & A & 0.94 & 2.06 & 0.45 & 248.0 & -0.025 \\
\hline & B & 0.94 & 1.57 & 0.35 & 246.4 & -0.021 \\
\hline & C & 0.94 & 2.05 & 0.43 & 252.7 & -0.029 \\
\hline & D & 0.95 & 1.98 & 0.52 & 252.9 & -0.025 \\
\hline \multirow[t]{4}{*}{3} & A & 1.64 & 1.49 & 0.52 & 150.7 & 0.010 \\
\hline & B & 1.65 & 1.33 & 0.54 & 176.5 & 0.018 \\
\hline & C & 1.17 & 1.88 & 0.60 & 223.5 & -0.008 \\
\hline & $D$ & 1.36 & 1.90 & 0.60 & 176.5 & 0.011 \\
\hline \multirow[t]{4}{*}{4} & A & 2. 10 & 0.35 & 0.21 & 120.0 & 0 \\
\hline & B & 2.06 & $1.2]$ & 0.35 & 219.5 & 0 \\
\hline & C & 2.14 & 0.90 & 0.54 & 224.9 & 0 \\
\hline & D & 2.40 & 0.67 & 0.38 & 226.4 & -0.007 \\
\hline \multirow[t]{4}{*}{5} & A & 1.92 & 0.61 & 0.16 & 137.5 & 0.018 \\
\hline & B & 2.12 & 0.59 & 0.21 & 180.0 & 0 \\
\hline & C & 1.80 & 1.05 & 0.20 & 198.4 & -0.043 \\
\hline & D & 1.80 & 0.53 & 0.15 & 201.7 & -0.033 \\
\hline
\end{tabular}


sine function, where $\mathrm{p}$ expresses the initial angular velocity and $r$ determines the change of this velocity. If $r$ is positive, the angular velocity increases and the figural process accelerates. If it is negative, the angular velocity decreases and the process decelerates. For $r=0$, the velocity will be constant.

The initial phase $\alpha$, which lasts from the onset of the trial to the first point in time where $s_{a}-s_{b}=0$ (i.e., the values for both part-figures are equal), differs from the other phases in some respects: (a) During the initial phase the subject is waiting and does not know which of the two alternating part-figures, the right or the left, will appear first. After the initial phase the two part-figures alternate regularly. (b) The first portion of the initial phase consists of a very short time, a fraction of a second, during which the subject seems to be unable to report any part-figure at all. The subsequent response phases of the observed fluctuations
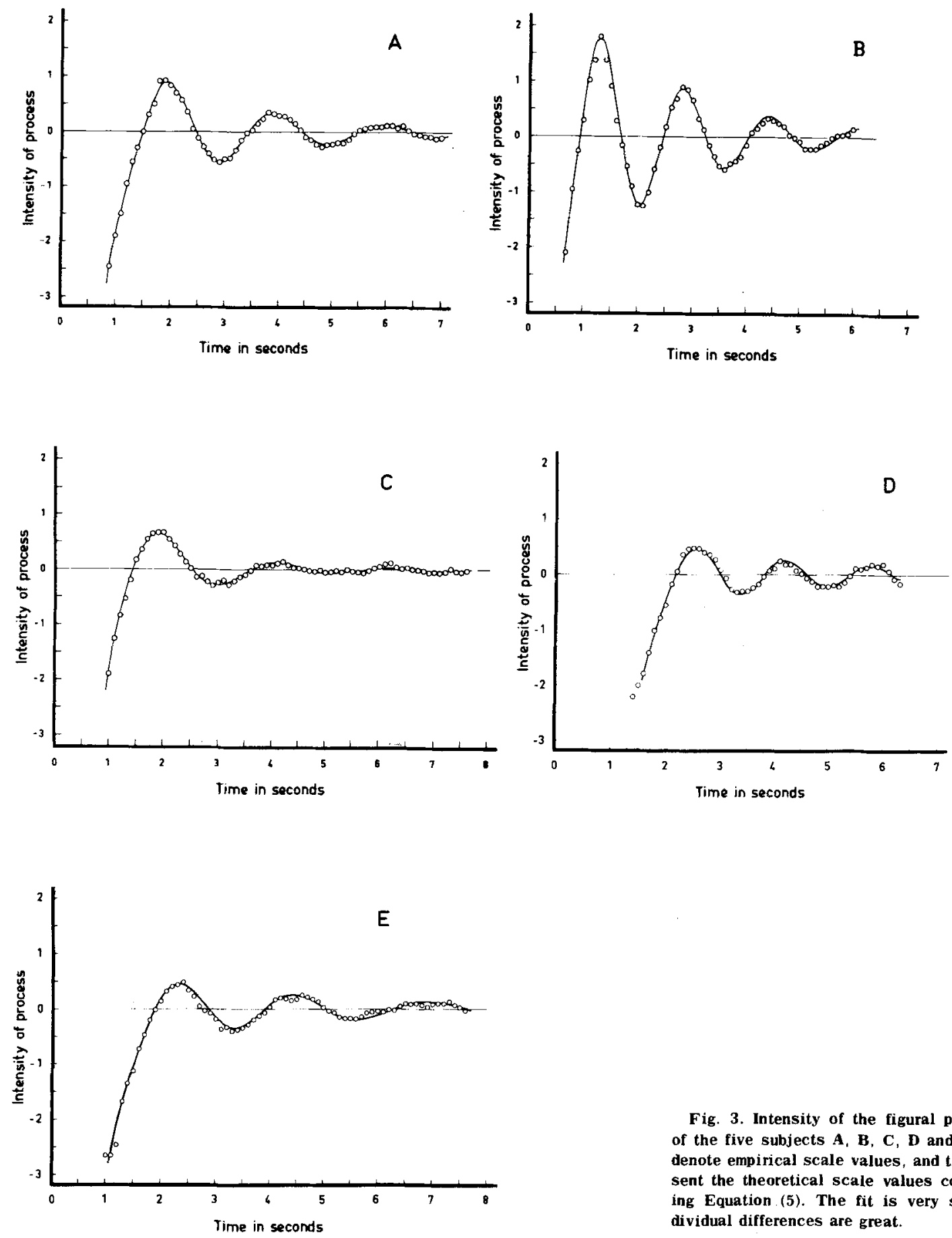

Fig. 3. Intensity of the figural process for each of the five subjects $A, B, C, D$ and $E$. The circles denote empirical scale values, and the curves represent the theoretical scale values computed according Equation (5). The fit is very satisfactory. Individual differences are great. 
Table 3 . Individual parameters.

\begin{tabular}{cccccr} 
Subject & $\alpha$ & A & q & \multicolumn{1}{c}{$p$} & \multicolumn{1}{c}{$r$} \\
\hline A & 1.49 & 1.09 & 0.46 & 187.8 & -0.008 \\
B & 0.94 & 2.09 & 0.49 & 248.0 & -0.019 \\
C & 1.45 & 1.01 & 0.80 & 170.5 & -0.008 \\
D & 2.18 & 0.53 & 0.33 & 219.0 & 0.007 \\
E & 1.90 & 0.50 & 0.26 & 193.5 & -0.038 \\
\hline
\end{tabular}

do not show a similar delay. (c) The remainder of the initial phase shows a rapid increase up to the level of $\mathrm{y}_{0}$. The succeeding proper phases increase and decrease periodically around $y_{0}$ 。

Considering the differing nature of the initial phase, Equation (5) is proposed to measure the intensity of the figural process following the initial phase. The initial phase will be discussed in a forthcoming report.

\section{RESULTS}

The individual parameters for the right and left part-figures are shown in Table 1. It is seen that there are no great differences, for any subject, between the right and left part-figures.

The individual results for each of four stimulus patterns are shown in Table 2. It may be seen that there are no great differences, for any subject, also between the four stimulus patterns.

Therefore all patterns and both part-figures may be treated together for each subject. The results are thus based on 280 records for each scale value. The psychological scale values are plotted over the time axis in Fig. 3. The graphs thus show the intensity of the figural process as measured by the method outlined above for all five subjects (A, B, C, D, E). The curves represent the theoretical values computed according to Equation (5). The circles denote empirical values. The fit is very satisfactory.

The individual parameters are shown in Table 3 for each subject. It is seen that the values of amplitude and frequency vary over individuals within rather wide limits.

\section{CONCLUSIONS}

(1) The main purpose of the present study was methodological: to apply an indirect scaling method to an experimental situation involving an observable but in practice not quantitatively reportable variation of perceptual intensity.

(2) As judged from the conspicuous regularity of computed scale values and the close agreement between empirical and theoretical values, the proposed scaling method appears well adapted to the kind of data obtained in the present experiments on figural fluctuations. It thus appears to be a useful instrument for the study of the intensity of the underlying figural process.

(3) One way of continuing this work would be to derive theoretical functions on the basis of psychological and/or physiological models, and to test hypothetical functions by fitting them to data obtained by the suggested method for scaling the intensity of the figural process.

\section{References}

Björkman, M. Measurement of learning. Stockholm: Almquist \& Wiksell, 1958.

Cohen, L. Rate of apparent change of a Necker cube as a function of prior stimulation. Amer. J. Psychol., 1959, 72, 327-344.

Ekman, G. Discriminal sensitivity on the subjective continuum. Acta Psychol., 1956, 12, 233-243.

Ekman, G. Weber's law and related functions. J. Psychol., 1959 , 47, 343-352.

Ekman, G., \& Künnapas, T. Measurement of aesthetic value by "direct" and "indirect" methods. Scand. J. Psychol., 1962a. 3, 33-39.

Ekman, G., \& Künnapas, T. Scales of aesthetic value. Percept. mot. Skills, $1962 \mathrm{~b}, 14,19-26$.

Ekman, G., \& Kunnapas, T. A further study of direct and indirect scaling methods. Scand. J. Psychol., 1963, 4, 77-80.

Ekman, G., Lindman, R. Measurement of the underlying process in perceptual fluctuations. Vision Res., 1962, 2, 253-260.

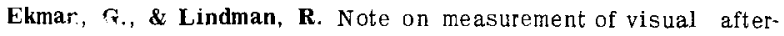
image intensity as a function of time. Vision Res., 1964, 4, 579-584.

Heath, H. A., Ehrlich, D., \& Orbach, J. Reversibility of the Necker cube: II. Effects of various activity conditions. Percept. mot. Skills, 1963, 17, 539-546.

Howard, I. P: An investigation of a satiation process in the reversible perspective of revolving skeletal shapes. Quart. J. exp. Psychol., 1961, 13, 19-33

Kolers, P. A. Apparent movement of a Necker cube. Amer. J. Psychol, 1964, 77, 220-230.

Kùnnapas, T. Experiments on figural dominance. J. exp. Psychol. $1957,53,31-39$.

Kunnapas, T. Measurement of the intensity of an underlying figural process. Scand. J. Psychol., 1961, 2, 174-184.

Olson, R., \& Orbach, J. Reversibility of the Necker cube: VIII. Parts of the figure contributing to the perception of reversals. Percept. mot. Skills, 1966, 22, 623-629.

Orbach, J., Ehrlich, D., \& Vainstein, E. Reversibility of the Necker cube: III. Effects of interpolation on reversal rate of the cube presented repetitively. Percept. mot. Skills. 1963, 17, 571-582.

Orbach, J., Zucker, E., \& Olson, R. Reversibility of the Necker cube: VII. Reversal tate as a function of figure-on and figure-off durations. Percept. mot. Skills, 1966, 22, 615-618.

oyama, $\boldsymbol{T}$. Figure-ground dominance as a function of sector angle, brightness, hue, and orientation. $J$. exp. Pszchol, 1960, 60, 299-305

Spitz, H. H., \& Lipman, R. S. Some factors affecting Necker cube reversal rate. Percept. mot. Skills, 1962, 15, 611-525.

Stevens, S. S. On the psychophysical law. Psychol. Rev., 1957, 64, 153-181.

Thetford, P. E. Influence of massing and spacing on Necker cube reversals. Percept. mot. Skills, 1963, 16, 215-222.

Thurstone, L. L. A law of comparative judgment. Psychol. Rev., 1927, 34, 273-286.

Torii, S. Figure-ground reversals under successively repeated observations, Jap. Psychol. Res, 1960, No. 9, 25-37.

\section{Note}

1. This investigation was supported by a research grant from the Swedish Council for Social Science Research. The writer is debted to Mrs. Monica Sillén and Mr. Udo Künnapas for their valuable assistance in rather laborious computational work.

(Received in the Editorial Office July 6, 1966.) 